\title{
ДЕСЯТЬ ЛУЧШИХ ИННОВАЦИОННЫХ ПРОДУКТОВ ВЫСТАВКИ VACUUMTECHEXPO 2018
}

\section{BEST INNOVATIVE PRODUCTS OF VACUUMTECHEXPO 2018}

DOI: $10.22184 / 1993-8578.2018 .84 .5 .312 .318$

\author{
C.Hecmepos* / sb.nesterov@vacuum.org.ru \\ S.Nesterov*
}

\begin{abstract}
Приведено описание десяти лучших инновационных продуктов выставки вакуумного оборудования "ВакуумТехЭкспо 2018".
\end{abstract}

The description of 10 best innovative products of the VacuumTechExpo 2018 is given in the paper.

2 4-26 апреля в Москве, в КВЦ "Сокольники", проходила XIII международная выставка вакуумного оборудования VacuumTechExpo 2018, организованная Международной группой компаний ITE при поддержке Российского научно-технического вакуумного общества им. академика С.А. Векшинского. В выставке приняли участие 70 компаний из 8 стран,

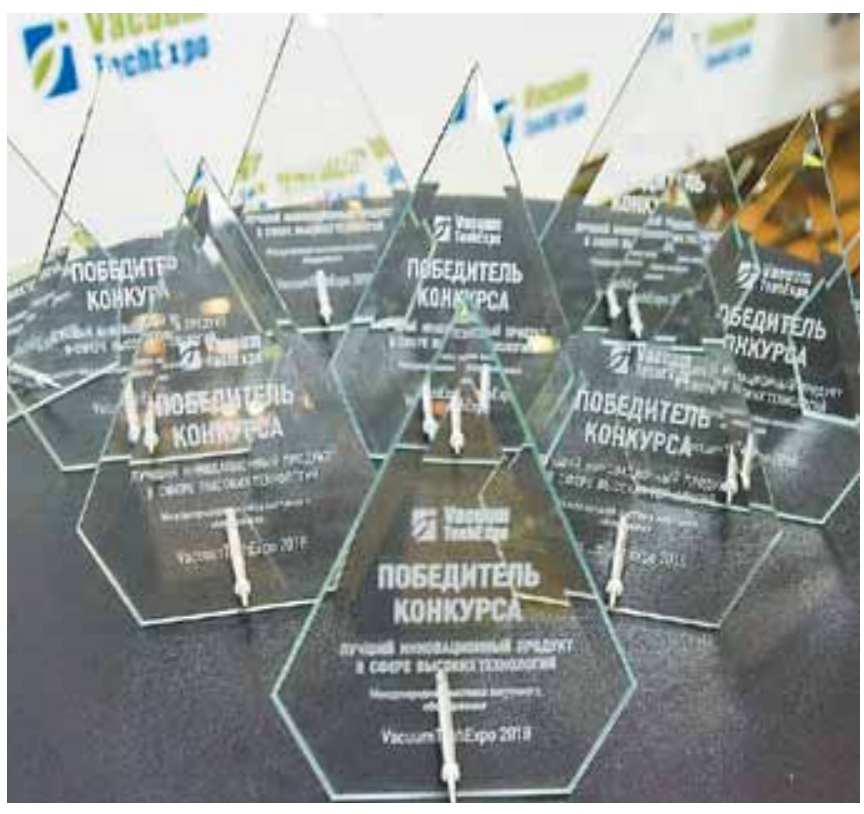

Памятные призы

Memorable prizes продемонстрировавшие вакуумные насосы и камеры, вакуумметры, преобразователи давления, вакуумную арматуру, установки для нанесения покрытий, вакуумные печи и сушильные шкафы, а также крионасосы, криоловушки и кулеры.

По традиции в ходе выставки проводился конкурс "Лучший инновационный продукт в сфере высоких технологий", победителями которого стали 10 продуктов - результатов инновационной деятельности, получивших практическую реализацию в виде нового товара, услуги, способа производства (технологии) или иного общественного результата. Представляем краткий обзор победителей, которые отражают современный уровень и тенденции развития мировой и отечественной вакуумной техники и технологии.

\section{КРИОГЕННЫЕ ВАКУУМНЫЕ НАСОСЫ \\ (ЗЭНКО ПЛАЗМА, МОСКВА)}

Криогенные вакуумные насосы ZP-DU предназначены для создания высокого безмасляного вакуума и обеспечивают высокие скорости откачки. В комплект поставки входят гелиевый компрессор, полимерные трубки для подачи гелия, кабель криоголовки, температурный контроллер с дисплеем и соединительными кабелями, нагревательная рубашка и клапан напуска. 


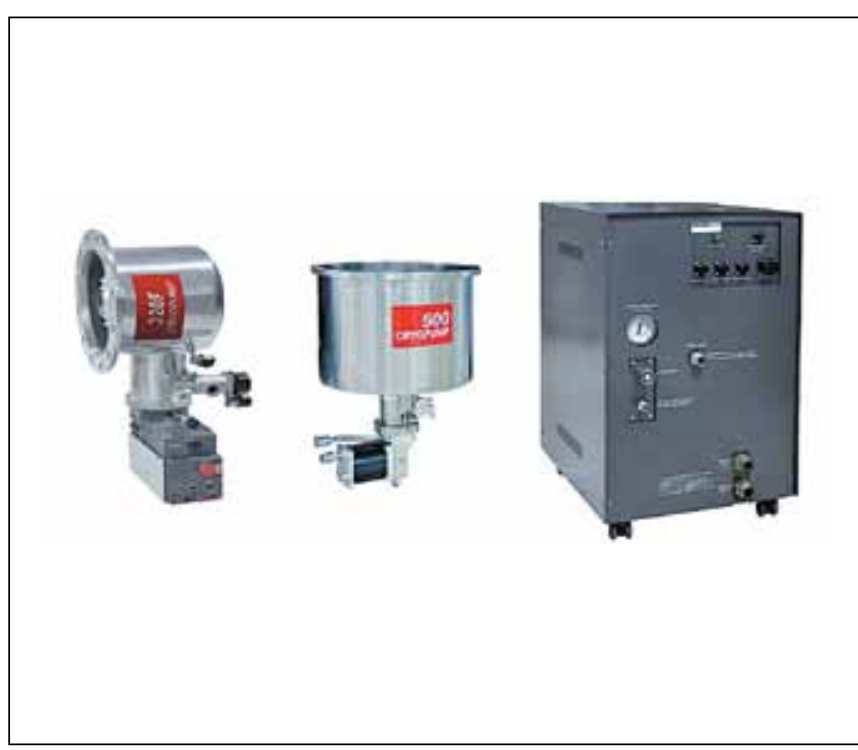

Криогенные вакуумные насосы ZP-DU

ZP-DU cryogenic vacuum pumps

Выпускаются следующие серии насосов:

- базовая серия - высокопроизводительные насосы с присоединительными фланцами DN200-DN500, обладающие очень хорошей устойчивостью к тепловому воздействию; радиальные элементы первой ступени насоса, конденсирующей пары воды, в отличие от поперечных элементов, обеспечивают лучшую проводимость $\mathrm{Ar} / \mathrm{N}_{2} / \mathrm{H}_{2}$, которые конденсируются на второй ступени насоса или поглощаются угольным фильтром;

- серия со встроенным контроллером, который позволяет полностью контролировать температуру захолаживания первой ступени, предотвращая "зависание" Ar;

- серия с эргономичным F-дизайном , характеризующаяся меньшими габаритными размерами;

- серия для производства жидкокристаллических дисплеев и органических светодиодов, выполнения вакуумного напыления и испарения. Увеличенные емкость второй ступени насоса и площадь криопанели позволяют конденсировать больше $\mathrm{Ar} / \mathrm{N}_{2}$ при увеличенном интервале между регенерациями.

Hacocы ZP-DU замещают аналогичные изделия из США и Японии, существенно дешевле последних и характеризуются низкими затратами на обслуживание благодаря наличию в Москве авторизованного сервисного центра. Реализованные технические решения защищены двумя международными патентами.

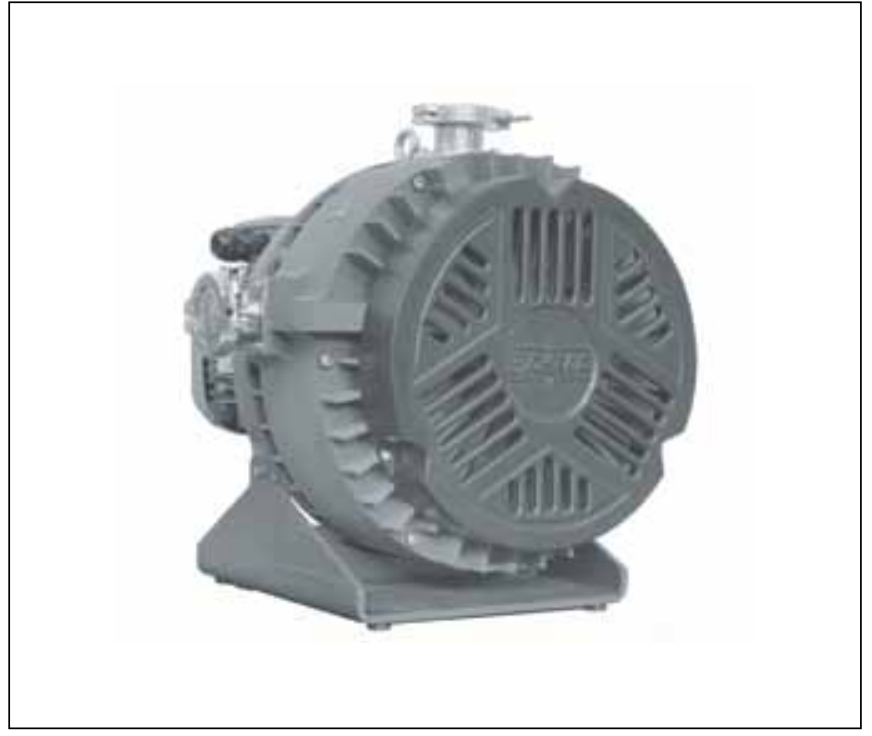

Вакуумный спиральный насос HBCn-60

HBCn-60 scroll vacuum pump

\section{ВАКУУМНЫЙ СПИРАЛЬНЫЙ НАСОС НВСП-60 (ВАКУУММАШ, КАЗАНЬ)}

НВСп-60 - новый вакуумный спиральный насос производительностью $60 \mathrm{~m}^{3} /$. Теперь первая серийно выпускающаяся в России серия насосов данного типа состоит из четырех моделей: НВСп-4, НВСп-12, НВСп-35 и НВСп-60.

Параметры НВСп-60:

- максимальная быстрота действия - $60 \pm 6 \mathrm{~m}^{3} / \mathrm{\varphi}$ $(16,7 \pm 1,7$ л/с);

- полное предельное остаточное давление - не более 1 Па без газобалластного устройства, 3 Па с газобалластным устройством;

- максимальное давление на входе при продолжительной работе - не более 10 кПа;

- масса - не более 56 кг с трехфазным электродвигателем или 58 кг с однофазным электродвигателем;

- габаритные размеры - $593 \times 424 \times 445$ мм с трехфазным электродвигателем или $510 \times 424 \times 445$ мм с однофазным электродвигателем;

- мощность электродвигателя - 1,5 кВт.

\section{СУХОЙ КУЛАЧКОВЫЙ ВАКУУМНЫЙ НАСОС СЕРИИ MINK MV (БУШ ВАКУУМ РУССИА, МОСКВА)}

Сухие кулачковые вакуумные насосы Mink MV являются усовершенствованием проверенной на практике серии Mink MM. Десятилетия опыта использования в различных областях позволили добиться существенного улучшения важнейших параметров насосов. Mink 


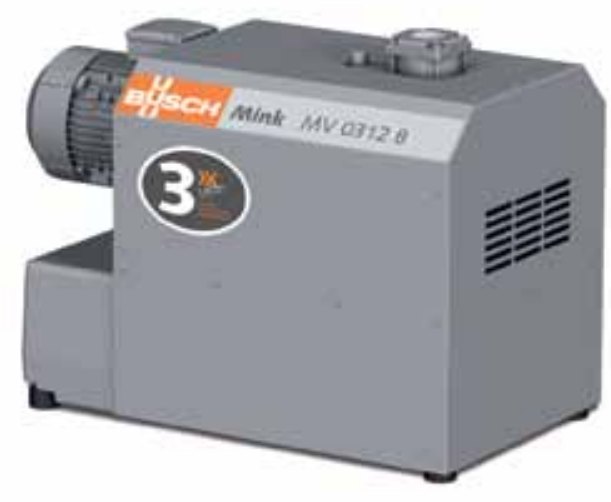

Кулачковый вакуумный насос Mink MV

Mink MV claw vacuum pump

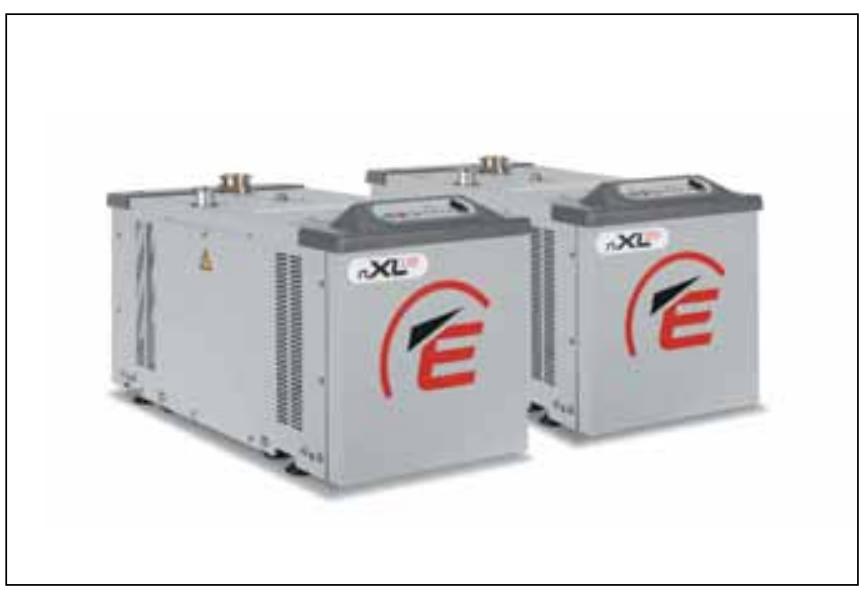

Вакуумные насосы с воздушным охиаждением Edwards nXLi Edwards $n X$ Li air cooled vacuum pumps

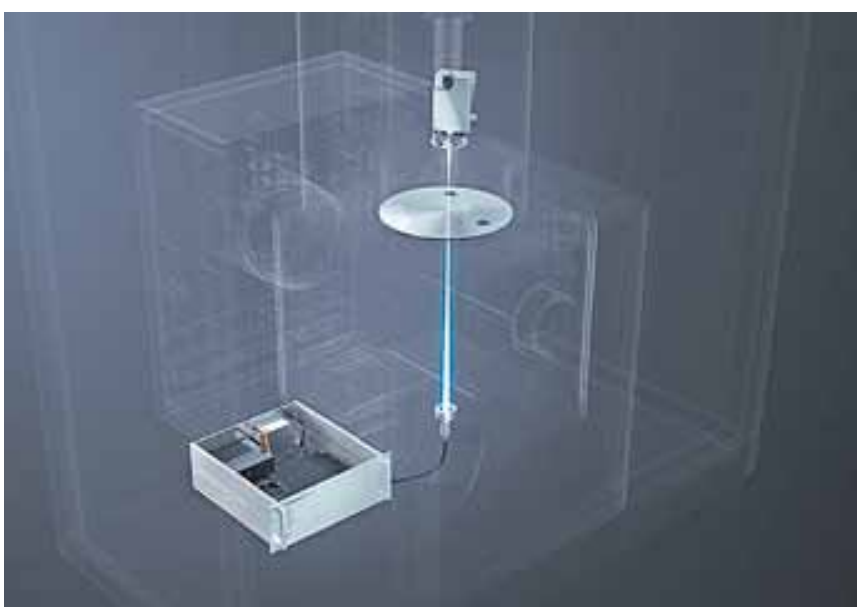

Комплекс оптического контроля тонкопленочных покрыmuŭ OCP Broadband

OCP Broadband system for optical control of thin-film coatings
MV работают чрезвычайно эффективно и способны на 60\% снизить суммарные эксплуатационные затраты по сравнению с традиционными решениями для получения вакуума. Благодаря компактным размерам насосы занимают мало места. Оптимизированная звукоизоляция позволила существенно снизить уровень шума.

\section{БЕЗМАСЛЯНЫЕ ОДНОФАЗНЫЕ НАСОСЫ СЕРИИ ПXLi (ИНТЕК АНАЛИТИКА, САНКТ-ПЕТЕРБУРГ)}

- Новая серия безмасляных спиральных насосов Edwards с производительностью до 200 м³ ч может применяться в научном машиностроении, масс-спектрометрии и других областях, требующих точности, управляемости и длительной надежности вакуумной откачки. Насосы nXLi имеют следующие преимущества:

- однофазное питание и воздушное охлаждение;

- управление - от ручного с передней панели до удаленного, в том числе цифрового по протоколу Modbus;

- минимальные габариты и уровень шума;

- встроенный обратный клапан на выхлопе для предотвращения обратного потока;

- безмасляный бесконтактный механизм обеспечивает максимальное время наработки на отказ (от 5 лет до первого сервисного обслуживания) при отсутствии необходимости обслуживания в межсервисный период;

- интеллектуальная система управления стартом и работой под нагрузкой обеспечивает максимальную производительность при откачке или возникновении газовой нагрузки при предельном вакууме и минимальное энергопотребление при стабилизированном вакууме.

\section{КОМПЛЕКС ОПТИЧЕСКОГО КОНТРОЛЯ ОСР BROADBAND (ИЗОВAК, МИНСК)}

Комплекс оптического контроля ОCP Broadband является легко интегрируемым решением для контроля процессов нанесения всех видов оптических покрытий на разных типах вакуумного технологического оборудования в полностью автоматическом режиме с разрешением до 0,3 нм. Особенность системы заключается в том, что программа управления анализирует реальный спектр покрытия в тесной связке с рассчитанным рецептом и определяет точку остановки для каждого слоя. 


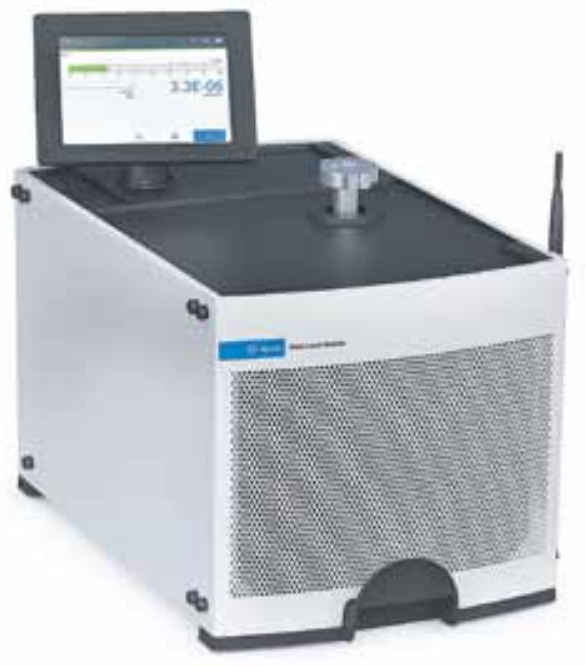

Гелиевый течеискатель Agilent HLD

Agilent HLD helium leak detector

Интеграция с контроллерами вакуумной установки позволяет реализовать автоматический процесс напыления покрытия. При необходимости программа может проводить оптимизацию расчета последующих слоев и изменять рецепт в онлайн-режиме онлайн для получения наилучшего совпадения сформированного покрытия с расчетным.

Отличительной особенностью ОСР Broadband является возможность работать как по пропусканию, так и по отражению, а также с неподвижным или перемещающимся свидетелем. Основной тип контроля - на пропускание непосредственно по детали или свидетелю, расположенному на движущемся по кругу подложкодержателе.

Широкополосная система автоматизированного оптического контроля ОСР Broadband может быть установлена как на оптические напылительные установки производства "Изовак", так и на вакуумную технику других производителей со стандартными протоколами обмена данными типа OPC server, Inficon IC6, Modbus и др.

В базовом спектральном диапазоне 370-1050 нм обеспечивается спектральное разрешение не хуже 0,5 нм при точности выставления длины волны не менее 0,2 нм и воспроизводимости 0,1 нм. Это позволяет с высокой точностью контролировать сложные структуры типа ультратонких узкополосных фильтров с полушириной до 1 нм или отрезающих фильтров с крутизной нарастания или спада до 1 нм. Еще одним преимуществом системы является возможность расширения диапазона контроля в ультрафиолетовую область до 220 нм либо в инфракрасную область спектра до 1650 нм, причем в УФ-области 220370 нм спектральное разрешение достигает 0,3 нм, а в ИК-диапазоне применяются спектрометры на базе IGA-линеек, разрешение которых ограничено 3 нм.

\section{ГЕЛИЕВЫЙ ТЕЧЕИСКАТЕЛЬ AGILENT HLD (МИЛЛАБ, МОСКВА)}

Главной особенностью нового течеискателя серии HLD является большой дисплей (21 cM), который имеет полностью обновленный интуитивно понятный пользовательский интерфейс с восемью доступными языками. Правильно настроить прибор позволяют шесть различных руководств, благодаря которым пользователи экономят время как на настройке, так и на испытании течеискателя.

Компания Agilent самостоятельно разрабатывает и производит все основные комплектующие для своих течеискателей, включая откачную систему и масс-спектрометр. Течеискатели Agilent совместимы как с лабораторными сверхвысоковакуумными установками, так и с большими промышленными системами.

Преимущества течеискателей Agilent:

- время отклика от 0,5 с;

- высокая точность благодаря расположению щели масс-спектрометра под углом $135^{\circ}$ в месте схождения пучка ионов гелия;

- возможность определять течи до 200 мбар;

- запатентованная система форвакуумных насосов у сухих течеискателей обеспечивает независимость от фона гелия;

- надежная система клапанов;

- простое двухкнопочное управление;

- низкий уровень шума.

\section{ВАКУУМНЫЕ МАГНИТОРАЗРЯДНЫЕ НАСОСЫ НМДК (КРИОСИСТЕМЫ, МОСКВА)}

Вакуумные магниторазрядные насосы НМдК предназначены для получения высокого и сверхвысокого вакуума при безмасляной откачке газов и газовых смесей. Новое поколение магниторазрядных 


\section{CONFERENCES, SEMINARS, EXHIBITIONS}

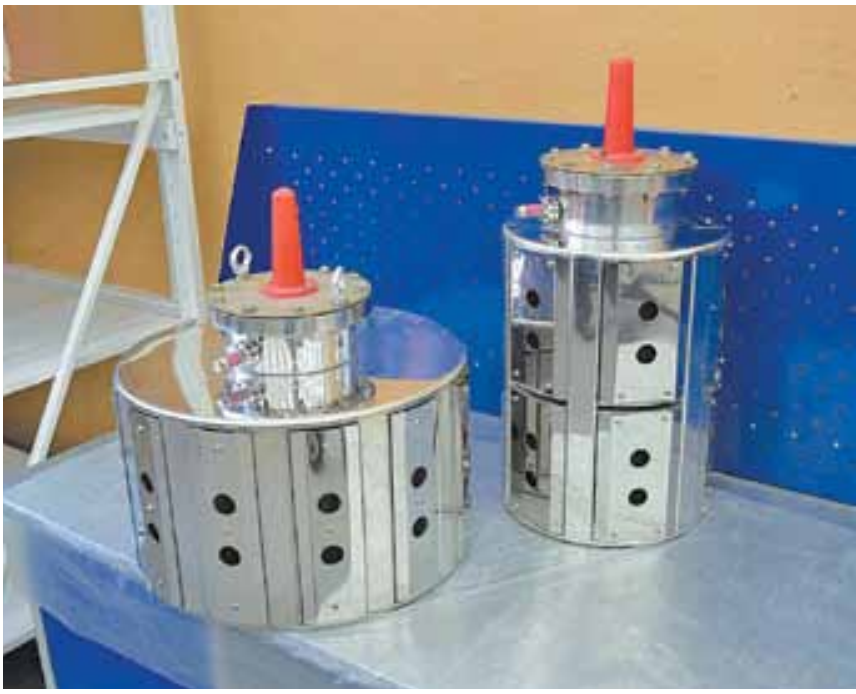

Вакуумные магниторазрядные насосы НМДК

Vacuum ion pumps

насосов производится в России ГК "Криосистемы" совместно с партнерами и является дальнейшим развитием отечественных насосов НМД.

Преимущества НМДК:

- простота конструкции внутренних частей насоса с минимальным количеством крепежных элементов;

- цилиндрическая форма позволила на 20-25\% снизить вес по сравнению с насосом НМД аналогичной производительности;

- современная магнитная система обеспечивает минимальные паразитные "вывалы" магнитного поля;

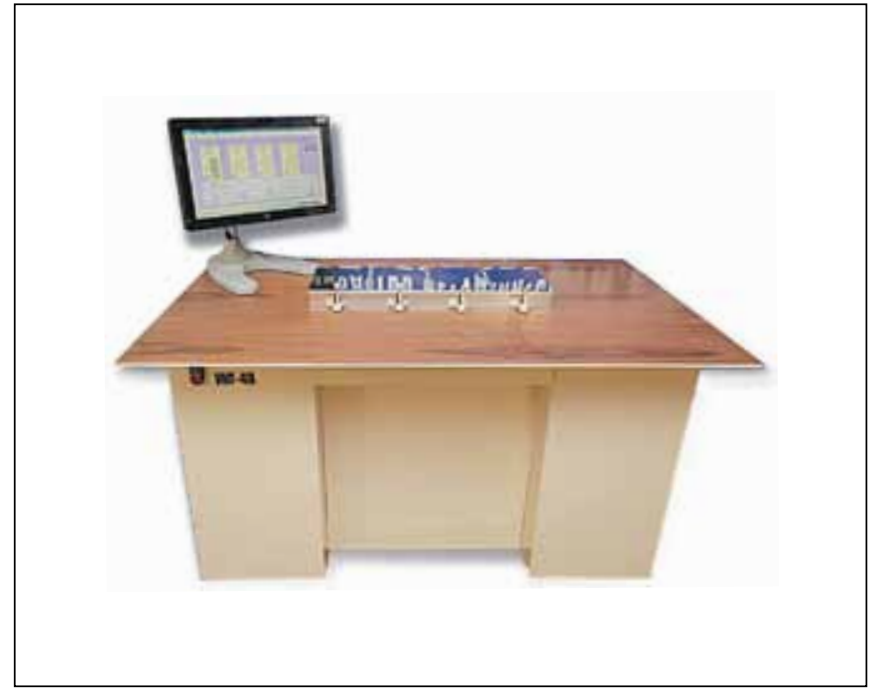

Установка масс-спектрометрического контроля герметичности УКГ-4П

Mass-spectrometric leak testing system

- конические элементы магнитной системы, плотно прилегая к карманам насоса, обеспечили на 5-10\% большую напряженность магнитного поля;

- ресурс работы при давлении $5 \cdot 10^{-4}$ Па составляет 50 тыс. ч;

- возможность размещения двух насосов в одном корпусе для удвоения производительности;

- тщательная очистка поверхностей и вакуумный отжиг всех элементов при температуре $450-680^{\circ} \mathrm{C}$ обеспечивают контролируемый состав газов внутри насоса и возможность довести предельный вакуум до $5 \cdot 10^{-9}$ Па; 


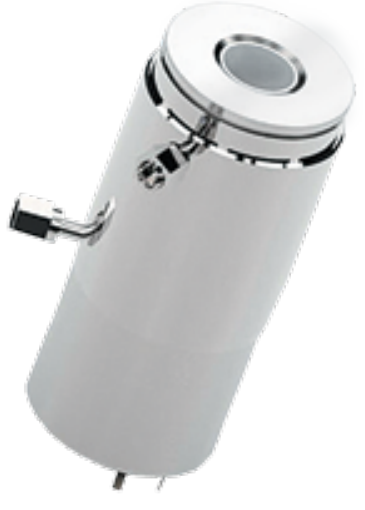

Водоохиаждаемый низкотемпературный источник испарения Hydravap

Hydravap water cooled low temperature evaporator

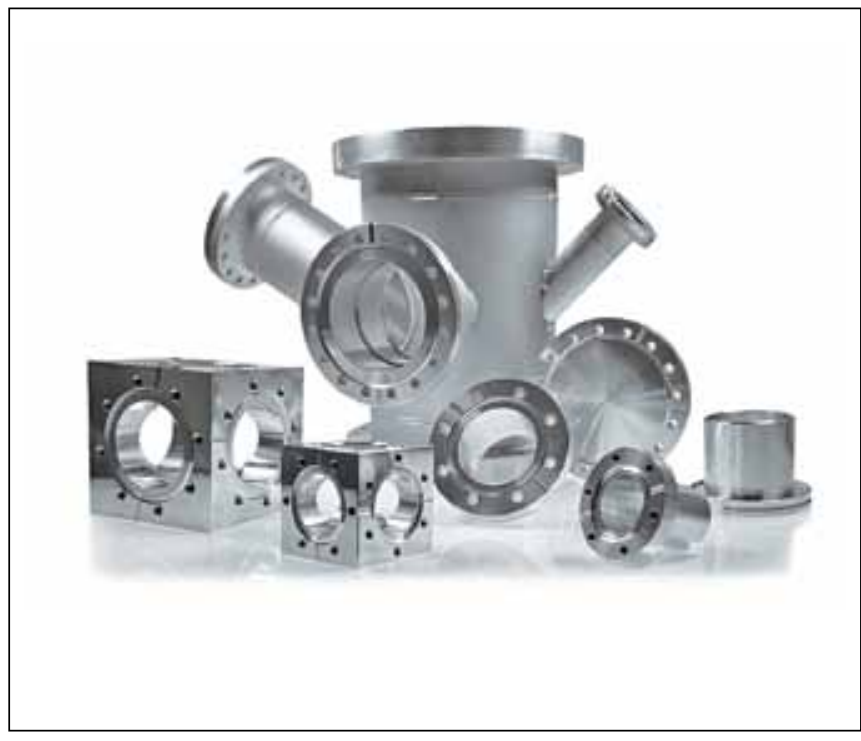

Алюминиевые вакуумные камеры и арматура Aluminum chambers and components

- токоввод насоса CF 16 согласуется с разъемами отечественных блоков питания байонетного типа старых и новых разработок.

\section{УСТАНОВКА КОНТРОЛЯ ГЕРМЕТИЧНОСТИ ДЛЯ ПОТОЧНЫХ ПРОИЗВОДСТВ ЭЛЕКТРОННОЙ ПРОМЫШЛЕННОСТИ (НПФ "ПРОГРЕСС", САНКТ-ПЕТЕРБУРГ)}

Установка масс-спектрометрического контроля герметичности УКГ-4П адаптирована к задачам производства электронных компонентов и состоит из четырех или более вакуумных камер, высоковакуумного безмасляного поста и масс-спектрометрической части с собственной высоковакуумной откачкой, смонтированных в виде моноблока размером $1100 \times 1140 \times 90$ см.

Оператор программно задает число задействованных в работе пеналов и минимальное время подготовки. Информация о работе установки и результат контроля каждого пенала отображается на мониторе. Стартом контроля последующего пенала является закрытие крышки загружаемого пенала. Пеналы снабжены световой индикацией, а при превышении установленного значения браковочного признака раздается звуковой сигнал.

Вес установки составляет около 100 кг, потребляемая мощность - менее 2 кВт.

\section{HYDRAVAP - ВОДООХЛАЖДАЕМЫЙ НИЗКОТЕМПЕРАТУРНЫЙ ИСТОЧНИК ИСПАРЕНИЯ ОРГАНИЧЕСКИХ МАТЕРИАЛОВ \\ (ЭЛЕКТРЕЙД-М, МОСКВА)}

Компания "Электрейд-M" в качестве официального партнера Kurt.J.Lesker Company представила новый водоохлаждаемый низкотемпературный источник испарения, предназначенный для применения в современных системах нанесения тонких пленок и покрытий методом вакуумного осаждения органических материалов на подложках. Основными преимуществами источника испарения Hydravap являются высокие скорость осаждения, качество и равномерность покрытия, точный контроль температуры и хорошая воспроизводимость параметров.

\section{АЛЮМИНИЕВЫЕ ВАКУУМНЫЕ КАМЕРЫ И APMATУPA (VACOM VAKUUM COMPONENTEN \& MESSTECHNIK GMBН, ГЕРМАНИЯ)}

Компания VACOM представила алюминиевые вакуумные камеры и арматуру с Conflatфланцами, в том числе четырехугольные камеры размером до $1200 \times 700 \times 600$ мм, цилин дрические камеры диаметром до 400 мм, специальные камеры с ConFlat-соединениями, а также фланцы разных типов и переходники. Все продукты совместимы с Conflatкомпонентами из нержавеющей стали.

$$
* * *
$$

Не вызывает сомнений, что выставка "ВакуумТехЭкспо" будет и дальше содействовать развитию российской инноватики. 